\title{
Neutralino pair production at proton-proton collider *
}

\author{
Han Liang ${ }^{a, b}$, Ma Wen-Gan ${ }^{a, b}$, Jiang Yi ${ }^{a}$, Zhou Mian-Lai ${ }^{a}$ and Zhou Hong ${ }^{a}$ \\ ${ }^{a}$ Department of Modern Physics, University of Science and Technology \\ of China (USTC), Hefei, Anhui 230027, China. \\ ${ }^{b}$ Institute of Theoretical Physics, Academia Sinica, \\ P.O.Box 2735, Beijing 100080, China.
}

\begin{abstract}
In this paper we investigated the Drell-Yan process of the light neutralino pair $\tilde{\chi}_{i}^{0} \tilde{\chi}_{j}^{0}(i, j=1,2)$ productions at hadron colliders. We studied the dependence of the coupling properties of two light neutralino $\tilde{\chi}_{1,2}^{0}$ on the three SUSY Lagrangian parameters $M_{1}, M_{2}$ and $\mu$, and find that the production rate of $\tilde{\chi}_{1}^{0} \tilde{\chi}_{2}^{0}$ pair will be dominated by the Higgsino-like couplings under the condition $\mu \ll M_{2}$ and $\mu \sim M_{1}$, while the productions of $\tilde{\chi}_{1}^{0} \tilde{\chi}_{1}^{0}$ and $\tilde{\chi}_{2}^{0} \tilde{\chi}_{2}^{0}$ pairs will be enhanced by the gaugino-like couplings under $M_{i} \ll \mu$. For the Higgsino-like $\tilde{\chi}_{1,2}^{0}$ neutralinos, the cross section of $\tilde{\chi}_{1}^{0} \tilde{\chi}_{2}^{0}$ production at the LHC can reach $100 f b$.
\end{abstract}

PACS number(s): 14.80.Ly, 12.60.Jv

\footnotetext{
${ }^{*}$ The project supported by National Natural Science Foundation of China
} 


\section{Introduction}

With various theoretically appealings, the Minimal Supersymmetric Standard Model (MSSM) is considered as the most potential choice for new physics beyond the Standard Model(SM). However, due to the absence of supersymmetric particle discovery in the energy range of current colliders, the supersymmetry (SUSY) is clearly not an exact symmetry of nature, and therefore must be broken. If R-parity conservation is hold and the SUSY breaking is around $\mathrm{TeV}$ scale, the first two neutralinos $\tilde{\chi}_{1,2}^{0}$ are expected to be light, and the lightest one $\tilde{\chi}_{1}^{0}$ is probably the lightest supersymmetric particle(LSP) in the SUSY models. Neutralinos would be the most promising particles for the first experimental SUSY test. Due to the high luminosity and large energy, the Large Hadron Collider(LHC) has the ability to produce neutralino pairs $\tilde{\chi}_{i}^{0} \tilde{\chi}_{j}^{0}$. In proton-proton collisions, $\tilde{\chi}_{i}^{0} \tilde{\chi}_{j}^{0}$ pairs can be produced via Drell-Yan subprocess and gluon-gluon fusion. Although the anti-quark luminosity in distribution function of proton is much lower than gluon, yet the cross sections of the neutralino pair productions via Drell-Yan mechanism are competitive with those from the gluon-gluon fusion, since the former mechanism of neutralino pair productions are accessible at the tree level. These facts make the production rates in Drell-Yan process are competitive with or even larger than those in gluon-gluon fusions. Therefore, it is significant in theoretical and experimental studies of $\tilde{\chi}_{i}^{0} \tilde{\chi}_{j}^{0}$ pair productions via $q \bar{q}$ collisions at LHC.

There are four SUSY model parameters involved in chargino/neutralino(ino) sector, i.e. gaugino mass parameter $M_{1}$ and $M_{2}$, Higgs mass parameter $\mu$ and the ratio of vacuum expectation values(VEV) $\tan \beta=v_{2} / v_{1}$. These four parameters are essential ingredients 
of the model: the two gaugino mass terms quantify the supersymmetry soft breaking of $S U(2) \times U(1)$ subgroup, and together with $\mu$ and $\tan \beta$ determine all the phenomenology properties in the ino sector. In order to extract the ino physical quantities (i.e. masses, mixings and couplings) from experimental observables (such as cross sections, branching ratios and L-R asymmetries), one needs to study thoroughly the relations of these quantities and observables with the fundamental SUSY parameters. The discussion of the pair productions of neutral gaugino and Higgsino current eigenstates can be found in Ref.[1]. However, the discussions were not based on physical neutralino particles, and thus could not really provide the sufficient information for the investigation of SUSY particles. In this work, we studied three typical pair production processes of neutralino mass eigenstates in proton-proton collisions, and investigated the dependence of their cross sections on SUSY Lagrangian parameters. The paper is organized as follows: in the section below, we present some general discussions on the diagonalization of ino mass matrixes. Sec. III gives the analytical tree-level formula of the cross sections of subprocesses $q \bar{q} \rightarrow \tilde{\chi}_{i}^{0} \tilde{\chi}_{j}^{0}$. In Sec.IV, we study the production rates of $\tilde{\chi}_{i}^{0} \tilde{\chi}_{j}^{0}$ pairs in both the subprocesses and the parent processes, and discuss the dependence of the production rates on the SUSY model parameters. Finally, a brief summary is presented.

\section{The MSSM parameters in chargino/neutralino sector}

The charginos $\tilde{\chi}_{j}^{+}(j=1,2)$ mass matrix in the current eigenstate basis has the form

$$
X=\left(\begin{array}{cc}
M_{2} & \sqrt{2} \sin \beta m_{W} \\
\sqrt{2} \cos \beta m_{W} & |\mu| e^{i \phi_{\mu}}
\end{array}\right)
$$


and the neutralino $\tilde{\chi}_{i}^{0}(i=1,4)$ mass matrix is

$$
Y=\left(\begin{array}{cccc}
M_{1} e^{i \phi_{1}} & 0 & -m_{Z} \sin \theta_{W} \cos \beta & m_{Z} \sin \theta_{W} \sin \beta \\
0 & M_{2} & m_{Z} \cos \theta_{W} \cos \beta & -m_{Z} \cos \theta_{W} \sin \beta \\
-m_{Z} \sin \theta_{W} \cos \beta & m_{Z} \cos \theta_{W} \cos \beta & 0 & |\mu| e^{i \phi_{\mu}} \\
m_{Z} \sin \theta_{W} \sin \beta & -m_{Z} \cos \theta_{W} \sin \beta & |\mu| e^{i \phi_{\mu}} & 0
\end{array}\right) .
$$

Without loss of any generality, $\mu$ and $M_{1}$ can be complex which can introduce CP-violation, and $M_{2}$ would be set to real and positive for its phase angle can be rotated away by the field redefinition and thus absorbed into $\phi_{\mu}$ and $\phi_{1}$. The diagonalizations of matrix $X$ and $Y$, which give all the ino masses and their couplings, can be achieved with

$$
\begin{aligned}
& U^{*} X V^{\dagger}=X_{D} \\
& N^{*} Y N^{\dagger}=Y_{D}
\end{aligned}
$$

In this work, we consider the ino sector with the following assumptions:

- For simplification, CP-conservation is hold, namely $\phi_{\mu}=\phi_{1}=0$.

- The physical signs among $M_{1}, M_{2}$ and $\mu$ are relative, which can be absorbed into phases $\phi_{\mu}$ and $\phi_{1}$ by redefinition of fields. Thus, $M_{1}, M_{2}$ and $\mu$ are chosen to be real and positive, i.e., $M_{1}, M_{2}, \mu>0$.

- $\tan \beta$ is always set as an input parameter, supposed that adequate information about $\tan \beta$ could be obtained from some other experiments beyond ino sector.

With the above assumptions, generally we have two ways to choose the input parameters in our calculation. One can employ the scenario of taking $M_{1}, M_{2}, \mu$ and $\tan \beta$ as input parameters, and then get all the physical ino masses and the matrix elements of $U, V$ and $N$ as 
outputs. The detail analyses can be found in Ref. [2] [3] [4]. Since the Lagrangian parameters should be extracted from the physical quantities, one can also choose an alternative way to diagonalize the mass matrix $Y$ by taking any three physical ino masses together with $\tan \beta$ as inputs. This strategy will provide the other three ino masses and all the mixings and couplings as outputs. There are several scenarios about the choices of three ino masses [5]. On the consideration that adequate information from chargino sector will give out the two mass values of $\tilde{\chi}_{1,2}^{+}$, and from the energy distribution of the final particles in the decay of $\tilde{\chi}_{1,2}^{+}$ at least one mass value of $m_{\tilde{\chi}_{i}^{0}}$ can be measured, in this paper we take two chargino masses $m_{\tilde{\chi}_{1,2}^{+}}$and one of the neutralino masses $m_{\tilde{\chi}_{i}^{0}}$ as inputs. In this way, the two fundamental SUSY parameter $M_{2}$ and $\mu$ can be figured out from the chargino masses by using following formula

$$
\left(M_{2}, \mu\right)=M_{ \pm}=\frac{1}{2}\left(\sqrt{\left(m_{\tilde{\chi}_{1}^{+}}+m_{\tilde{\chi}_{2}^{+}}\right)^{2}-2 m_{W}^{2}(1-\sin 2 \beta)} \pm \sqrt{\left(m_{\tilde{\chi}_{1}^{+}}-m_{\tilde{\chi}_{2}^{+}}\right)^{2}-2 m_{W}^{2}(1+\sin 2 \beta)}\right)
$$

The ambiguity in determining $\mu$ and $M_{2}$ from the two-fold values of $M_{ \pm}$can be cleaned up with some favourable measurements on the chargino phenomenology. Practically, if the behaviours of charginos and their couplings are Higgsino-like, there exists $M_{2} \gg \mu$, while if they are gaugino-like, we have $M_{2} \ll \mu$. As to $M_{1}$, it is usually a free parameter in the MSSM, if we have no further assumption. In the second input strategy, $M_{1}$ can be determined by one of neutralino masses $m_{\tilde{\chi}_{i}^{0}}$ alone, when $M_{2}$ and $\mu$ are given by Eq.(2.3).

$$
M_{1}=\frac{1}{2}\left\{\mp 2 m_{\tilde{\chi}_{i}^{0}}\left(m_{\tilde{\chi}_{i}^{0}}^{2}-\mu^{2}\right)\left(m_{\tilde{\chi}_{i}^{0}} \mp M_{2}\right) \pm 2 m_{\tilde{\chi}_{i}^{0}}^{2} m_{Z}^{2}-2 m_{\tilde{\chi}_{i}^{0}} M_{2} m_{Z}^{2} \sin ^{2} \theta_{W}+\right.
$$




$$
\begin{array}{r}
\left.\mu\left(2 m_{\tilde{\chi}_{i}^{0}} \mp M_{2}\right) m_{Z}^{2} \sin 2 \beta \pm \mu M_{2} m_{Z}^{2} \sin 2 \beta \cos 2 \theta_{W}\right\} / \\
\left\{\left(\mu^{2}-m_{\tilde{\chi}_{i}^{0}}^{2}\right)\left(m_{\tilde{\chi}_{i}^{0}} \mp M_{2}\right)+m_{Z}^{2} \cos ^{2} \theta_{W}\left(m_{\tilde{\chi}_{i}^{0}} \pm \mu \sin 2 \beta\right)\right\}
\end{array}
$$

Generally there are two solutions for $M_{1}$, and one is positive while the other is negative. With the assumptions mentioned above, we take the positive value solution from Eq.(2.4),

and fix $M_{1}$ definitely. Therefore, from the input mass values of two charginos $\left(m_{\chi_{1,2}^{+}}\right)$and one of the neutralino mass $\left(m_{\chi_{i}^{0}}\right)$, one can extract all the SUSY mass parameters $M_{1}$ and $M_{ \pm}$which denote $M_{2}$ and $\mu$ alternatively, and then figure out the mass spectra and all the couplings of neutralino sector consequently. In the following calculation and discussion, we will adopt both input strategies discussed above.

\section{Neutralino pair productions in $q \bar{q}$ collisions}

The Neutralino pair productions processes via the collisions of quark and anti-quark in protons, can be expressed as

$$
q\left(p_{1}\right) \bar{q}\left(p_{2}\right) \rightarrow \tilde{\chi}_{i}^{0}\left(k_{1}\right) \tilde{\chi}_{j}^{0}\left(k_{2}\right) \quad(i, j=1,2,3,4)
$$

where $p_{1}$ and $p_{2}$ represent the momenta of the incoming quark and anti-quark, and $k_{1}$ and $k_{2}$ denote the momenta of the two final state neutralinos, respectively. The Mandelstam variables $\hat{s}, \hat{t}$ and $\hat{u}$ are defined as $\hat{s}=\left(k_{1}+k_{2}\right)^{2}, \hat{t}=\left(p_{1}-k_{1}\right)^{2}, \hat{u}=\left(p_{1}-k_{2}\right)^{2}$. The relevant Feynmann diagrams are drawn in Fig.1. The interaction Lagrangians involved are listed as 
[6] [0]

$$
\begin{gathered}
\mathcal{L}_{Z q \bar{q}}=\frac{g}{\cos \theta_{W}} \cdot \bar{q} \gamma^{\mu}\left[a_{Z q}^{S}+a_{Z q}^{L} P_{L}\right] q Z_{\mu} \\
\mathcal{L}_{Z \chi^{0} \chi^{0}}=\frac{g}{2 \cos \theta_{W}} \cdot \bar{\chi}_{i}^{0} \gamma^{\mu}\left[O_{Z}^{i j} P_{L}-O_{Z}^{i j}{ }^{\star} P_{R}\right] \chi_{j}^{0} Z_{\mu} \\
\mathcal{L}_{q \tilde{q} \chi^{0}}=-\sqrt{2} g \cdot \bar{q}\left[a_{q k i}^{L} P_{L}+a_{q k i}^{R} P_{R}\right] \chi_{i}^{0} \tilde{q}_{k}
\end{gathered}
$$

with the coupling constants

$$
\begin{gathered}
a_{Z q}^{S}=Q_{q} \sin ^{2} \theta_{W}, \quad a_{Z q}^{L}=\frac{1}{2}(-1)^{T_{q}^{3}+1 / 2} \\
O_{Z}^{i j}=N_{i, 4} N_{j, 4}^{\star}-N_{i, 3} N_{j, 3}^{\star} \\
a_{q k i}^{L}=\frac{m_{q} N_{i, 5-q}^{\star}}{2 m_{W} \beta_{q}} R_{q k, 1}^{\star}-\tan \theta_{W} Q_{q} N_{i, 1}^{\star} R_{q k, 2}^{\star} \\
a_{q k i}^{R}=\left(T_{q}^{3} N_{i, 2}-\tan \theta_{W}\left(T_{q}^{3}-Q_{q}\right) N_{i, 1}\right) R_{q k, 1}^{\star}+\frac{m_{q} N_{i, 5-q}}{2 m_{W} \beta_{q}} R_{q k, 2}^{\star}
\end{gathered}
$$

Here $P_{R, L}=\left(1 \pm \gamma_{5}\right) / 2 . \quad R_{q}$ denote the squark transformation matrix. $q=1,2$ denote up-type and down-type quarks respectively, and

$$
\beta_{q}= \begin{cases}\sin \beta & q=1 \\ \cos \beta & q=2\end{cases}
$$

The corresponding Lorentz invariant matrix element for the tree-level process is written as

$$
\mathcal{M}_{0}=\mathcal{M}_{\hat{s}}+\mathcal{M}_{\hat{t}}+\mathcal{M}_{\hat{u}}
$$

where

$$
\mathcal{M}_{\hat{s}}=\frac{i g^{2}}{2 \cos ^{2} \theta_{W} \cdot\left(\hat{s}-m_{Z}^{2}\right)} \bar{u}\left(k_{1}\right) \gamma^{\mu}\left[O_{Z}^{i j} P_{L}-O_{Z}^{i j \star} P_{R}\right] v\left(k_{2}\right) \cdot \bar{v}\left(p_{2}\right) \gamma_{\mu}\left[a_{Z q}^{S}+a_{Z q}^{L} P_{L}\right] u\left(p_{1}\right)
$$




$$
\begin{gathered}
\mathcal{M}_{\hat{t}}=\frac{-2 i g^{2}}{\hat{t}-m_{\tilde{q}_{k}}^{2}} \bar{u}\left(k_{1}\right)\left(a_{q k i}^{L \star} P_{R}+a_{q k i}^{R \star} P_{L}\right) u\left(p_{1}\right) \cdot \bar{v}\left(p_{2}\right)\left(a_{q k j}^{L} P_{L}+a_{q k j}^{R} P_{R}\right) v\left(k_{2}\right) \quad(k=1,2) \\
\mathcal{M}_{\hat{u}}=(-1)^{\delta_{i j}} \frac{-2 i g^{2}}{\hat{u}-m_{\tilde{q}_{l}}^{2}} \bar{u}\left(k_{2}\right)\left(a_{q l j}^{L \star} P_{R}+a_{q l j}^{R \star} P_{L}\right) u\left(p_{1}\right) \cdot \bar{v}\left(p_{2}\right)\left(a_{q l i}^{L} P_{L}+a_{q l i}^{R} P_{R}\right) v\left(k_{1}\right) \quad(l=1,2)
\end{gathered}
$$

Here we take simply vanished masses of the first generation quarks, i.e. $m_{u}=m_{d}=0$. The relative $\operatorname{sign}(-1)^{\delta_{i j}}$ of $\mathcal{M}_{\hat{u}}$ towards $\mathcal{M}_{\hat{t}}$ and $\mathcal{M}_{\hat{s}}$ is merely due to Pauli statistics.

The corresponding differential cross section at tree level can be written as

$$
\frac{d \sigma}{d \Omega}=\frac{1}{4} \frac{N_{c}}{9}\left(\frac{1}{2}\right)^{\delta_{i j}} \frac{g^{4} \lambda_{i j}}{32 \pi^{2} \hat{s}^{2}}\left(I_{\hat{s} \hat{s}}+I_{\hat{t} \hat{t}}+I_{\hat{u} \hat{u}}+2 I_{\hat{s} \hat{t}}+2(-1)^{\delta_{i j}} I_{\hat{s} \hat{u}}+2(-1)^{\delta_{i j}} I_{\hat{t} \hat{u}}\right)
$$

where the factors $\frac{1}{4}, \frac{N_{c}}{9}$ and $\left(\frac{1}{2}\right)^{\delta_{i j}}$ are the initial spin-average, color-average and final identical-particle factors respectively. The squares of matrix element have the form as

$$
\begin{aligned}
& I_{\hat{s} \hat{s}}=\frac{2}{\cos ^{4} \theta_{W}\left(\hat{s}-m_{Z}^{2}\right)^{2}}\left(a_{Z q}^{S}{ }^{2}+a_{Z q}^{S} a_{Z q}^{L}+\frac{1}{2} a_{Z q}^{L}{ }^{2}\right) \\
& {\left[O_{Z}^{i j} O_{Z}^{i j \star} \cdot\left(\left(m_{\tilde{\chi}_{i}}^{2}-\hat{t}\right)\left(m_{\tilde{\chi}_{j}}^{2}-\hat{t}\right)+\left(m_{\tilde{\chi}_{i}}^{2}-\hat{u}\right)\left(m_{\tilde{\chi}_{j}}^{2}-\hat{u}\right)\right)-\left(O_{Z}^{i j 2}+O_{Z}^{i j \star 2}\right) \cdot m_{\tilde{\chi}_{i}} m_{\tilde{\chi}_{j}} \hat{s}\right]} \\
& I_{\hat{t} \hat{t}}=\frac{4}{\left(\hat{t}-m_{\tilde{q}_{k}}^{2}\right)\left(\hat{t}-m_{\tilde{q}_{k^{\prime}}}^{2}\right)}\left(a_{q k^{\prime} i}^{L} a_{q k i}^{L \star}+a_{q k^{\prime} i}^{R} a_{q k i}^{R \star}\right)\left(a_{q k j}^{L} a_{q k^{\prime} j}^{L \star}+a_{q k j}^{R} a_{q k^{\prime} j}^{R \star}\right) \cdot\left(m_{\tilde{\chi}_{i}}^{2}-\hat{t}\right)\left(m_{\tilde{\chi}_{j}}^{2}-\hat{t}\right) \\
& I_{\hat{u} \hat{u}}=\frac{4}{\left(\hat{u}-m_{\tilde{q}_{l}}^{2}\right)\left(\hat{u}-m_{\tilde{q}_{l^{\prime}}}^{2}\right)}\left(a_{q l^{\prime} j}^{L} a_{q l j}^{L \star}+a_{q l^{\prime} j}^{R} a_{q l j}^{R \star}\right)\left(a_{q l i}^{L} a_{q l^{\prime} i}^{L \star}+a_{q l i}^{R} a_{q l^{\prime} i}^{R \star}\right) \cdot\left(m_{\tilde{\chi}_{i}}^{2}-\hat{u}\right)\left(m_{\tilde{\chi}_{j}}^{2}-\hat{u}\right) \\
& I_{\hat{s} \hat{t}}=\frac{-2}{\cos ^{2} \theta_{W}\left(\hat{s}-m_{Z}^{2}\right)\left(\hat{t}-m_{\tilde{q}_{k}}^{2}\right)} \\
& {\left[\left(a_{Z q}^{S} a_{q k i}^{L} a_{q k j}^{L \star} O_{Z}^{i j}-\left(a_{Z q}^{S}+a_{Z q}^{L}\right) a_{q k i}^{R} a_{q k j}^{R \star} O_{Z}^{i j \star}\right) \cdot\left(m_{\tilde{\chi}_{i}}^{2}-\hat{t}\right)\left(m_{\tilde{\chi}_{j}}^{2}-\hat{t}\right)+\right.} \\
& \left.\left(\left(a_{Z q}^{S}+a_{Z q}^{L}\right) a_{q k i}^{R} a_{q k j}^{R \star} O_{Z}^{i j}-a_{Z q}^{S} a_{q k i}^{L} a_{q k j}^{L \star} O_{Z}^{i j \star}\right) \cdot m_{\tilde{\chi}_{i}} m_{\tilde{\chi}_{j}} \hat{s}\right]
\end{aligned}
$$




$$
\begin{aligned}
I_{\hat{s} \hat{u}}= & \frac{-2}{\cos ^{2} \theta_{W}\left(\hat{s}-m_{Z}^{2}\right)\left(\hat{u}-m_{\tilde{q}_{l}}^{2}\right)} \\
& {\left[\left(\left(a_{Z q}^{S}+a_{Z q}^{L}\right) a_{q l j}^{R} a_{q l i}^{R \star} O_{Z}^{i j}-a_{Z q}^{S} a_{q l j}^{L} a_{q l i}^{L \star} O_{Z}^{i j \star}\right) \cdot\left(m_{\tilde{\chi}_{i}}^{2}-\hat{u}\right)\left(m_{\tilde{\chi}_{j}}^{2}-\hat{u}\right)+\right.} \\
& \left.\left(a_{Z q}^{S} a_{q l j}^{L} a_{q l i}^{L \star} O_{Z}^{i j}-\left(a_{Z q}^{S}+a_{Z q}^{L}\right) a_{q l j}^{R} a_{q l i}^{R \star} O_{Z}^{i j \star}\right) \cdot m_{\tilde{\chi}_{i}} m_{\tilde{\chi}_{j}} \hat{s}\right] \\
I_{\hat{t} \hat{u}}= & \frac{4}{\left(\hat{t}-m_{\tilde{q}_{k}}^{2}\right)\left(\hat{u}-m_{\tilde{q}_{l}}^{2}\right)} \\
& {\left[\left(a_{q l i}^{L \star} a_{q k j}^{L} a_{q k i}^{L \star} a_{q l j}^{L}+a_{q l i}^{R \star} a_{q k j}^{R} a_{q k i}^{R \star} a_{q l j}^{R}\right) \cdot m_{\tilde{\chi}_{i}} m_{\tilde{\chi}_{j}} \hat{s}+\right.} \\
& \frac{1}{2}\left(a_{q l i}^{L \star} a_{q k j}^{L} a_{q k i}^{R \star} a_{q l j}^{R}+a_{q l i}^{R \star} a_{q k j}^{R} a_{q k i}^{L \star} a_{q l j}^{L}\right) \cdot \\
& \left.\left(\left(m_{\tilde{\chi}_{i}}^{2}-\hat{t}\right)\left(m_{\tilde{\chi}_{j}}^{2}-\hat{t}\right)+\left(m_{\tilde{\chi}_{i}}^{2}-\hat{u}\right)\left(m_{\tilde{\chi}_{j}}^{2}-\hat{u}\right)-\left(\hat{s}-m_{\tilde{\chi}_{i}}^{2}-m_{\tilde{\chi}_{j}}^{2}\right) \hat{s}\right)\right]
\end{aligned}
$$

where

$$
\lambda_{i j}=\sqrt{\left(\hat{s}-m_{\tilde{\chi}_{i}}^{2}-m_{\tilde{\chi}_{j}}^{2}\right)^{2}-4 m_{\tilde{\chi}_{i}}^{2} m_{\tilde{\chi}_{j}}^{2}} / 2
$$

\section{Numerical results and discussion}

As we assume $\tilde{\chi}_{1,2}^{0}$ are lighter than $\tilde{\chi}_{3,4}^{0}$ and $\tilde{\chi}_{1}^{0}$ is likely to be the LSP, the three types of channels: $q \bar{q} \rightarrow \tilde{\chi}_{1}^{0} \tilde{\chi}_{1}^{0}, q \bar{q} \rightarrow \tilde{\chi}_{2}^{0} \tilde{\chi}_{2}^{0}$ and $q \bar{q} \rightarrow \tilde{\chi}_{1}^{0} \tilde{\chi}_{2}^{0}$ (associated production) would be the most dominant neutralino pair production processes which may lead to the first detection of SUSY particles at the $\mathrm{LHC}[8]$. Here we present the numerical results of the cross sections of these three processes and discuss their dependences on the basic SUSY parameters. We divide the input MSSM parameters into two parts. One is for for the general parameters included also in the SM, and the other is the ino and squark sectors of the MSSM. For the first parameter 
part, we take $m_{Z}=91.1887 \mathrm{GeV}, \sin ^{2} \theta_{W}=0.2315, \alpha=1 / 137.03598$. For the second part, we just limit the values of $M_{1}, M_{2}$ and $\mu$ to be real, positive and below $1 \mathrm{TeV}$, and take $\tan \beta=1.5, m_{\tilde{u}_{1}}=m_{\tilde{d}_{1}}=350 \mathrm{GeV}, m_{\tilde{u}_{2}}=m_{\tilde{d}_{2}}=550 \mathrm{GeV}$, and $\theta_{\tilde{u}}=\theta_{\tilde{d}}=\pi / 4$. The three ino physical masses are taken as

$$
m_{\tilde{\chi}_{1}^{+}}=150 \mathrm{GeV}, \quad m_{\tilde{\chi}_{2}^{+}}=550 \mathrm{GeV}, \quad m_{\tilde{\chi}_{1}^{0}}=100 \mathrm{GeV}
$$

By using Eq.(2.3) with above ino mass values, one may have two choices of parameter sets for $\mu$ and $M_{2}$, which are in two extreme cases respectively, namely Higgsino-like and gaugino-like ino states. For the Higgsino-like case, we get

$$
M_{2}=534 \mathrm{GeV}, \quad \mu=166 \mathrm{GeV}, \quad M_{1}=135 \mathrm{GeV},
$$

and other neutralino masses as

$$
m_{\tilde{\chi}_{2}^{0}}=166 \mathrm{GeV}, \quad m_{\tilde{\chi}_{3}^{0}}=184 \mathrm{GeV}, \quad m_{\tilde{\chi}_{4}^{0}}=550 \mathrm{GeV} .
$$

For the gaugino-like case, we have

$$
M_{2}=166 \mathrm{GeV}, \quad \mu=534 \mathrm{GeV}, \quad M_{1}=105 \mathrm{GeV}
$$

and other three neutralino masses as

$$
m_{\tilde{\chi}_{2}^{0}}=151 \mathrm{GeV}, \quad m_{\tilde{\chi}_{3}^{0}}=534 \mathrm{GeV}, \quad m_{\tilde{\chi}_{4}^{0}}=554 \mathrm{GeV} .
$$

For complete discussion, we present also the results for the mixture case. We take the physical mass inputs as follows for the mixture ino states

$$
m_{\tilde{\chi}_{1}^{+}}=122 \mathrm{GeV}, \quad m_{\tilde{\chi}_{2}^{+}}=280 \mathrm{GeV}, \quad m_{\tilde{\chi}_{1}^{0}}=101 \mathrm{GeV}
$$


the corresponding outputs obtained as

$$
\begin{gathered}
M_{2}=\mu=200 \mathrm{GeV}, \quad M_{1}=150 \mathrm{GeV} \\
m_{\tilde{\chi}_{2}^{0}}=164 \mathrm{GeV}, \quad m_{\tilde{\chi}_{3}^{0}}=201 \mathrm{GeV}, \quad m_{\tilde{\chi}_{4}^{0}}=286 \mathrm{GeV},
\end{gathered}
$$

Then the cross sections of the subprocesses $q \bar{q} \rightarrow \tilde{\chi}_{1}^{0} \tilde{\chi}_{1}^{0}, \tilde{\chi}_{1}^{0} \tilde{\chi}_{2}^{0}, \tilde{\chi}_{2}^{0} \tilde{\chi}_{2}^{0}$ can be numerically evaluated. The cross sections for the three subprocesses are plotted in Fig.2(a),(b) and (c) respectively, as the functions of the parton-parton collision c.m.s. energy $\sqrt{\hat{s}}$. In Fig.2(a) and (c), all the curves for gaugino-like case show obviously the threshold effects when $\sqrt{\hat{s}}$ is just above the threshold energies of the $\tilde{\chi}_{1}^{0} \tilde{\chi}_{1}^{0}$ and $\tilde{\chi}_{2}^{0} \tilde{\chi}_{2}^{0}$ pair production, respectively. The cross sections for Higgsino-like case are fairly smaller than the corresponding ones for gaugino-like case in the process of $\tilde{\chi}_{i}^{0} \tilde{\chi}_{i}^{0}$ pair production, and the curves for the Higgsino-like $\tilde{\chi}_{2}^{0} \tilde{\chi}_{2}^{0}$ pair production are even too small to be plotted in Fig.2(c). On the contrary, the Higgsino couplings will enhance abruptly the cross sections for the processes of $\tilde{\chi}_{1}^{0} \tilde{\chi}_{2}^{0}$ pair production as shown in Fig.2(b), but the cross sections for gaugino-like and mixture cases are negligibly small compared with those for Higgsino-like case.

The reactions of $q \bar{q} \rightarrow \tilde{\chi}_{i}^{0} \tilde{\chi}_{j}^{0}$ are only subprocesses of the parent $p p$ hadron collider. The total cross sections of $\tilde{\chi}_{i}^{0} \tilde{\chi}_{j}^{0}$ pair productions via $q \bar{q}$ annihilation in $p p$ collider can be simply obtained by folding the cross sections of the subprocesses $\hat{\sigma}\left[q \bar{q} \rightarrow \tilde{\chi}_{i}^{0} \tilde{\chi}_{j}^{0}\right] \quad(q=u, d)$ with the quark and anti-quark luminosity. Adopting quark and anti-quark structure functions of the MRS set $\mathrm{G}$ given in Ref. [9] and $Q=\sqrt{\hat{s}}$, we calculate the cross sections $\sigma[p p \rightarrow$ $\left.q \bar{q} \rightarrow \tilde{\chi}_{i}^{0} \tilde{\chi}_{j}^{0}\right](i, j=1,2)$ as the functions of the $p p$ collider c.m.s energy $\sqrt{s}$, under the same 
conditions of Eq.(4.1). The results are depicted in Fig.2(d). It is impressive that the $\tilde{\chi}_{1}^{0} \tilde{\chi}_{2}^{0}$ pair production rate at the $\mathrm{LHC}$, can even reach as large as $1.5 \times 10^{2} \mathrm{fb}$, when the couplings are Higgsino-like. We also calculate the $\tilde{\chi}_{1}^{0} \tilde{\chi}_{2}^{0}$ pair production at the Tevatron. The cross section $\sigma\left[p \bar{p} \rightarrow q \bar{q} \rightarrow \tilde{\chi}_{1}^{0} \tilde{\chi}_{2}^{0}\right]$ can be $0.02 p b$ in Higgsino-like case, when $\sqrt{s}=2 \mathrm{TeV}$. We see that the light neutralino pair production rates at the Tevatron are far smaller than those at the LHC, due to the lower c.m.s energy at Tevatron.

In Fig.3(a),(b) and (c), the cross sections $\hat{\sigma}\left[u \bar{u} \rightarrow \tilde{\chi}_{1}^{0} \tilde{\chi}_{1}^{0}, \tilde{\chi}_{1}^{0} \tilde{\chi}_{2}^{0}, \tilde{\chi}_{2}^{0} \tilde{\chi}_{2}^{0}\right]$ versus the parameter $M_{2}$, with $\sqrt{\hat{s}}=1 \mathrm{TeV}$ and $\mu=400 \mathrm{GeV}$, are plotted by taking $M_{1}=100,400 \mathrm{GeV}$ and $1 \mathrm{TeV}$, respectively. As discussed above with the physical masses $m_{\tilde{\chi}_{1,2}^{+}}$and one of $m_{\tilde{\chi}_{i}^{0}}$, one might get three model parameters $M_{1}$ and $M_{ \pm}$where $M_{ \pm}$denote $M_{2}$ and $\mu$ alternatively. The properties of neutralinos depend not only on $M_{2}$ and $\mu$ as charginos do, but also on the mass parameter $M_{1}$. From Fig.3, we can say in the case that when there is a large split between $M_{ \pm}\left(M_{+} \gg M_{-}\right)$, the $\tilde{\chi}_{i}^{0} \tilde{\chi}_{j}^{0}$ pair productions via $q \bar{q}$ collisions have some interesting features as follows:

- When $M_{1} \gg M_{ \pm}, \tilde{\chi}_{1,2}^{0}$ are mainly decide by $M_{2}$ and $\mu$ : if $\mu=M_{-} \ll M_{2}=M_{+}, \tilde{\chi}_{1,2}^{0}$ are dominantly composed of Higgsino, and consequently $\tilde{\chi}_{1}^{0} \tilde{\chi}_{2}^{0}$ pair production rate is significant while $\tilde{\chi}_{i}^{0} \tilde{\chi}_{i}^{0} \quad(i=1,2)$ pair productions are suppressed; if $\mu=M_{-} \gg M_{2}=$ $M_{+}, \tilde{\chi}_{1}^{0}$ is approximately $\tilde{W}_{3}$ and $\tilde{\chi}_{2}^{0}$ is Higgsino, and accordingly $\tilde{\chi}_{1}^{0} \tilde{\chi}_{1}^{0}$ pair production is enhanced while $\tilde{\chi}_{1}^{0} \tilde{\chi}_{2}^{0}$ and $\tilde{\chi}_{2}^{0} \tilde{\chi}_{2}^{0}$ pair productions are suppressed. Shown as in Fig.3 with the cases of $M_{1}=1 \mathrm{TeV}$, and the curve of $\tilde{\chi}_{2}^{0} \tilde{\chi}_{2}^{0}$ pair production for the case $M_{1}=1 \mathrm{TeV}$ is too small to be plotted in Fig.3(c). 
- When $M_{1} \ll M_{ \pm}, \tilde{\chi}_{1}^{0}$ is $\tilde{B}$. Accordingly, $\tilde{\chi}_{1}^{0} \tilde{\chi}_{1}^{0}, \tilde{\chi}_{1}^{0} \tilde{\chi}_{2}^{0}$ pair productions are dominated by gaugino-couplings, and the former is enhance and the latter is suppressed (the exceptation for the curve of $M_{1}=100 \mathrm{GeV}$ in Fig.3(b) are merely because of the mass degeneration between $m_{\tilde{\chi}_{1,2}^{0}}$ when $\left.M_{1}=M_{2}=100 \mathrm{GeV}\right)$. $\tilde{\chi}_{2}^{0}$ are decide by $M_{2}$ and $\mu$ : if $M_{2}=M_{-} \ll \mu=M_{+}, \tilde{\chi}_{2}^{0}$ is mainly $\tilde{W}_{3}$, and $\tilde{\chi}_{2}^{0} \tilde{\chi}_{2}^{0}$ pair production is enhanced; and vice versa. Shown as in Fig. 3 with the cases of $M_{1}=100 \mathrm{GeV}$.

- When $M_{-}<M_{1}<M_{+}$, the effects of $M_{1}$ on $\tilde{\chi}_{1,2}^{0}$ are rather weak, and only lead to some mixture. Then if $\mu=M_{-} \ll M_{2}=M_{+}, \tilde{\chi}_{1,2}^{0}$ are in some Higgsino-like states, and consequently $\tilde{\chi}_{1}^{0} \tilde{\chi}_{2}^{0}$ pair production is significant while $\tilde{\chi}_{i}^{0} \tilde{\chi}_{i}^{0}(\mathrm{i}=1,2)$ pairs are suppressed; if $M_{2}=M_{-} \ll \mu=M_{+}, \tilde{\chi}_{1}^{0}$ is in gaugino-like state while $\tilde{\chi}_{2}^{0}$ may be in some mixture state, and accordingly $\tilde{\chi}_{1}^{0} \tilde{\chi}_{1}^{0}$ pair production is enhanced while $\tilde{\chi}_{1}^{0} \tilde{\chi}_{2}^{0}, \tilde{\chi}_{2}^{0} \tilde{\chi}_{2}^{0}$ pairs are suppressed. Shown as in Fig.3 with the cases of $M_{1}=400 \mathrm{GeV}$.

These features can be concluded as that pure gaugino-couplings dominate the production of $\tilde{\chi}_{i}^{0} \tilde{\chi}_{i}^{0}$ pair (as shown by the curves in the areas $M_{2}<\mu=400 \mathrm{GeV}$ of Fig.3(a,c)), while pure Higgsino couplings enhance the $\tilde{\chi}_{i}^{0} \tilde{\chi}_{j}^{0}(i \neq j)$ pair productions (as shown by the curve of $M_{1}=1 \mathrm{TeV}$ in the area $\mu \ll M_{2}$ of Fig.3(b)), and any gaugino-like $\tilde{\chi}_{1}^{0}$ will spoil the large production rate of $\tilde{\chi}_{1}^{0} \tilde{\chi}_{2}^{0}$.

\section{Conclusions}

In this work we studied the productions of the light neutralino pairs $\tilde{\chi}_{1}^{0} \tilde{\chi}_{1}^{0}, \tilde{\chi}_{1}^{0} \tilde{\chi}_{2}^{0}, \tilde{\chi}_{2}^{0} \tilde{\chi}_{2}^{0}$ via Drell-Yan process at hadron colliders, and investigated the correlations between the property 
of the neutralino pair productions and the basic SUSY Lagrangian parameter $M_{1}, M_{2}$ and $\mu$.

From the numerical results, it can be concluded that Higgsino-couplings will increase the $\tilde{\chi}_{1}^{0} \tilde{\chi}_{2}^{0}$ pair production significantly, while gaugino-couplings enhance the $\tilde{\chi}_{1}^{0} \tilde{\chi}_{1}^{0}$ and $\tilde{\chi}_{2}^{0} \tilde{\chi}_{2}^{0}$ pair productions. If $\mu<<M_{2}$ and $\mu_{\sim}^{<} M_{1}$, Higgsino components will be dominated in $\tilde{\chi}_{1}^{0}$ and $\tilde{\chi}_{2}^{0}$, and the cross section of Higgsino-like $\tilde{\chi}_{1}^{0} \tilde{\chi}_{2}^{0}$ pair production at the LHC, can reach $100 f b$. Thus, by taking a annual luminosity of $p p$ collision at the LHC being $100 f b^{-1}$, one can accumulate $1 \times 10^{4}$ events per year. Therefore the precise measurement of this process is suitable in detecting SUSY signals and helpful in determining the basic SUSY parameters.

Acknowledgement: These work was supported in part by the National Natural Science Foundation of China(project numbers: 19675033, 19875049), the Youth Science Foundation of the University of Science and Technology of China and a grant from the State Commission of Science and Technology of China.

\section{References}

[1] J. Ellis and Graham G. Ross, Phys. Lett. B117(1982)397; J.M. Frere and G.L. Kane, Nucl. Phys. B223(1983)331.

[2] Mohamed M.El Kheishen, Ahmed A. Shafik and Amr A. Aboshousha, Phys. Rev. D45(1992)4345-4345.

[3] M.L. Zhou, W.G. Ma, L. Han, Y. Jiang and H. Zhou, J. Phys. G25(1999)27-43 
[4] S.Y. Choi, A. Djouadi, H.S. Song and P.M. Zerwas, hep-ph/9812236.

[5] J.L. Kneur and G. Moultaka, hep-ph/9807336.

[6] J.F. Gunion and H.E. Haber, Nucl. Phys. B272 (1986)1-76.

[7] J. Rosiek, Phys. Rev. D41(1998)3464.

[8] H.E. Haber and G.L. Kane, Phys. Rep. 117 (1985)75-263.

[9] A.D. Martin, R.G. Roberts and W.J.Stirling, Phys. Lett. B354(1995)155-162.

\section{Figure Captions}

Fig. 1 The Feynman diagrams of the subprocess $q \bar{q} \rightarrow \tilde{\chi}_{i}^{0} \tilde{\chi}_{j}^{0}$.

Fig.2(a) The cross sections of the subprocess $q \bar{q} \rightarrow \tilde{\chi}_{1}^{0} \tilde{\chi}_{1}^{0}$ as functions of $\sqrt{\hat{s}}$. The letter 'u' and 'd' denote the different processes with initial $u \bar{u}$ and $d \bar{d}$ collisions respectively, while 'H', 'g' and 'm' denote the Higgsino-like, gaugino-like and mixture cases respectively.

Fig.2(b) The cross sections of the subprocess $q \bar{q} \rightarrow \tilde{\chi}_{1}^{0} \tilde{\chi}_{2}^{0}$ as functions of $\sqrt{\hat{s}}$. The letter 'u' and 'd' denote the different processes with initial $u \bar{u}$ and $d \bar{d}$ collisions respectively, while 'H', 'g' and 'm' denote the Higgsino-like, gaugino-like and mixture cases respectively.

Fig.2(c) The cross sections of the subprocess $q \bar{q} \rightarrow \tilde{\chi}_{2}^{0} \tilde{\chi}_{2}^{0}$ as functions of $\sqrt{\hat{s}}$. The letter 'u' and 'd' denote the different processes with initial $u \bar{u}$ and $d \bar{d}$ collisions respectively, while 'H', 'g' and 'm' denote the Higgsino-like, gaugino-like and mixture cases respectively.

Fig.2(d) The total cross sections of the process $p p \rightarrow q \bar{q} \rightarrow \tilde{\chi}_{i}^{0} \tilde{\chi}_{j}^{0}$ as functions of $\sqrt{s}$, where 'H', 'g' and 'm' denote the Higgsino-like, gaugino-like and mixture cases, respectively. 
Fig.3(a) The cross sections of the subprocess $u \bar{u} \rightarrow \tilde{\chi}_{1}^{0} \tilde{\chi}_{1}^{0}$ as functions of $M_{2}$ with $\mu=$ $400 \mathrm{GeV}$ and $\sqrt{\hat{s}}=1 \mathrm{TeV}$.

Fig.3(b) The cross sections of the subprocess $u \bar{u} \rightarrow \tilde{\chi}_{1}^{0} \tilde{\chi}_{2}^{0}$ as functions of $M_{2}$ with $\mu=$ $400 \mathrm{GeV}$ and $\sqrt{\hat{s}}=1 \mathrm{TeV}$.

Fig.3(c) The cross sections of the subprocess $u \bar{u} \rightarrow \tilde{\chi}_{2}^{0} \tilde{\chi}_{2}^{0}$ as functions of $M_{2}$ with $\mu=$ $400 \mathrm{GeV}$ and $\sqrt{\hat{s}}=1 \mathrm{TeV}$. 


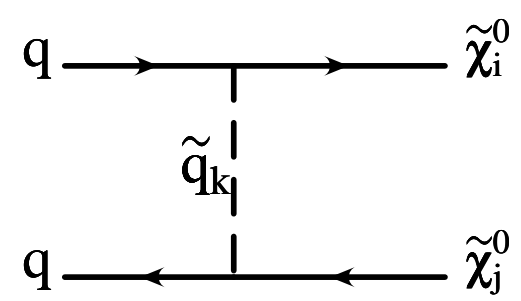

Fig1(a1)

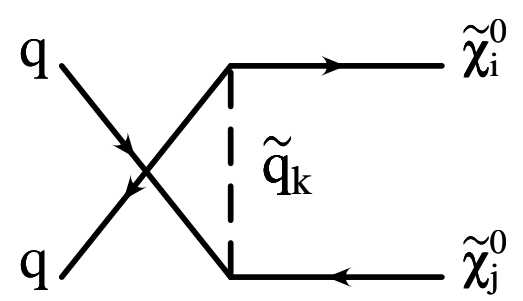

Fig1(a2)

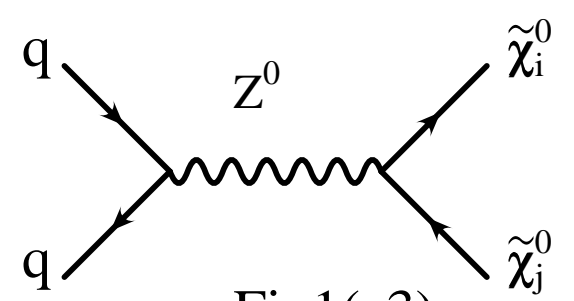

Fig1(a3) 
Fig.2(a)

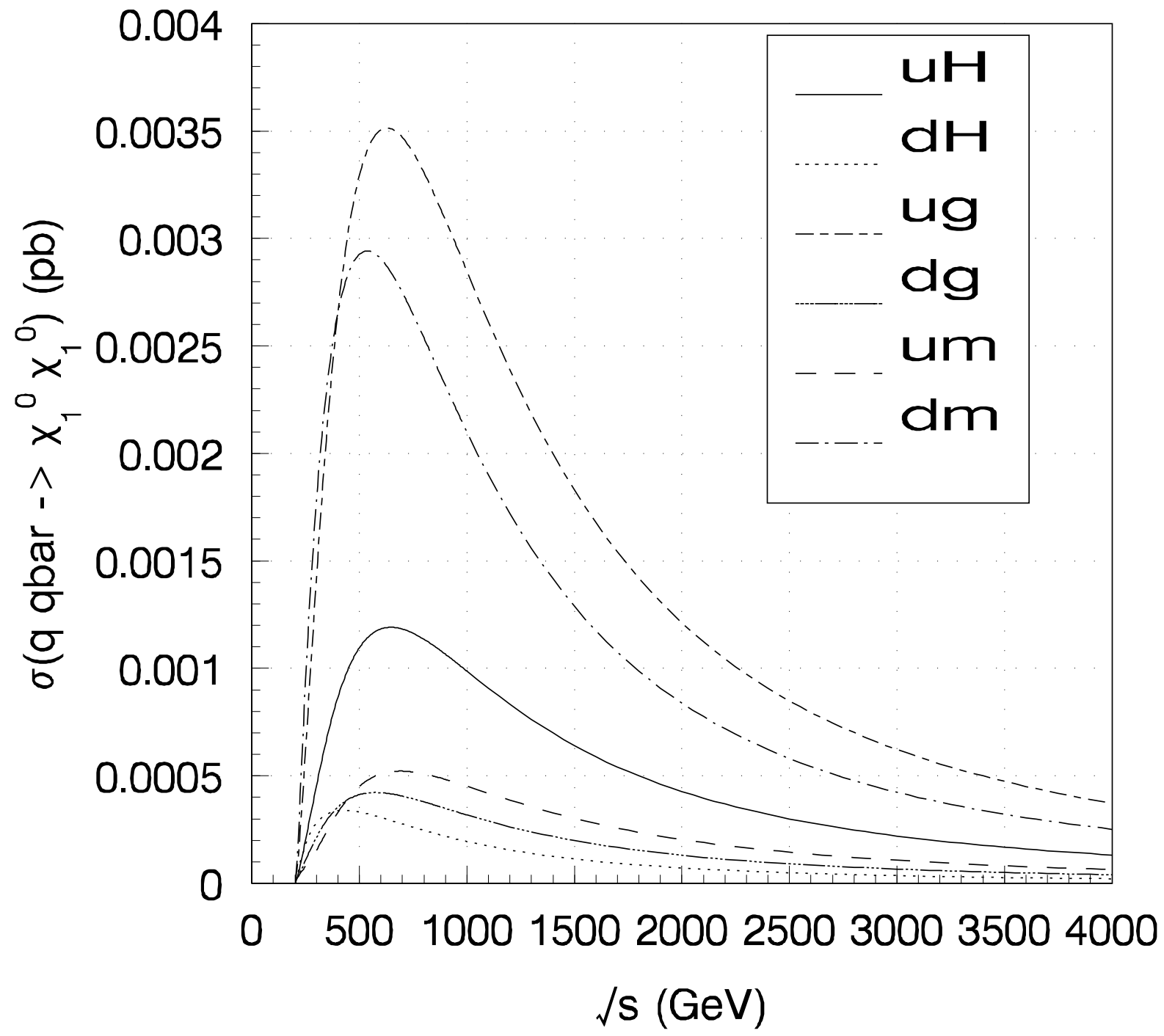


Fig.2(b)

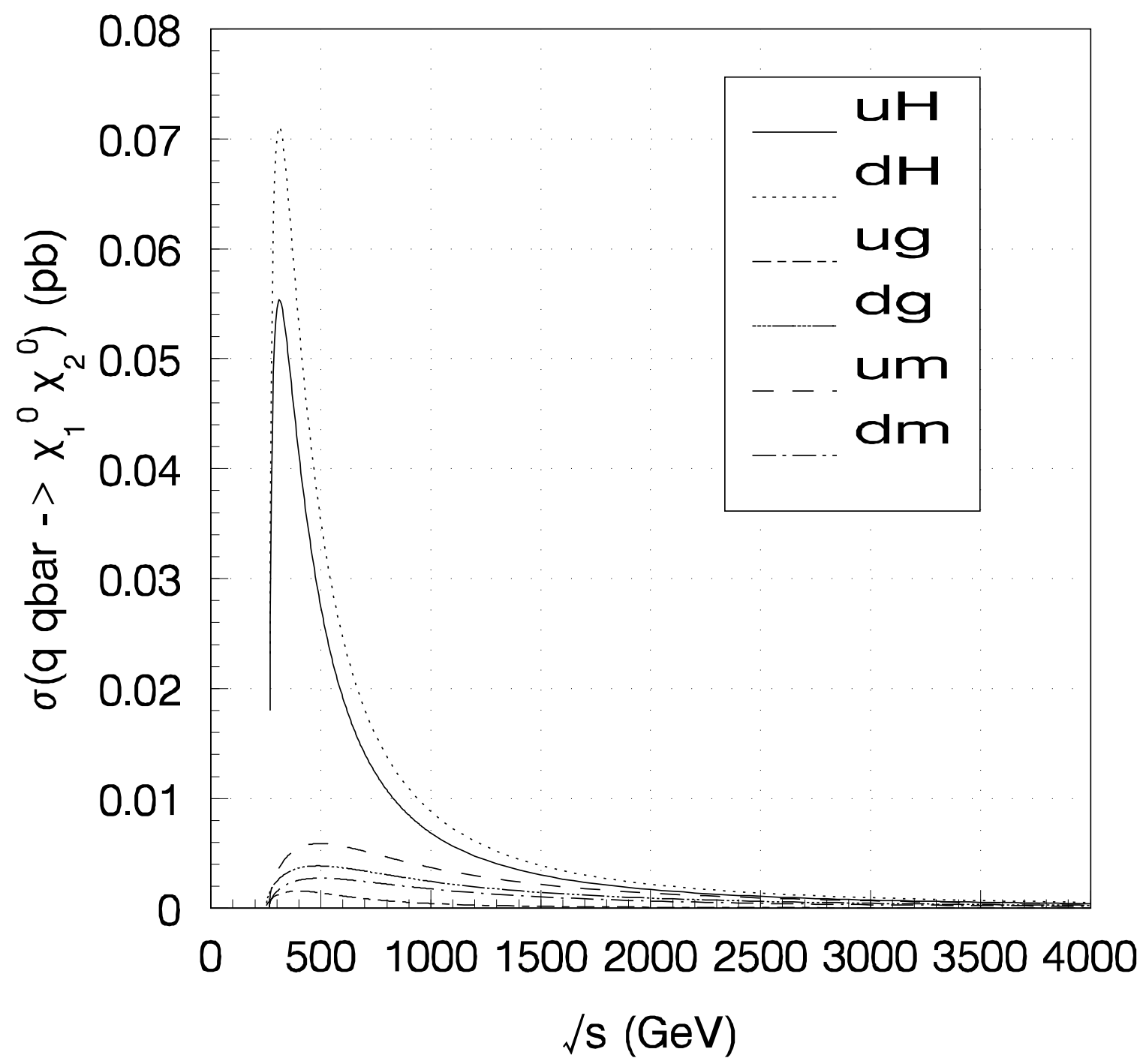


Fig.2(c)

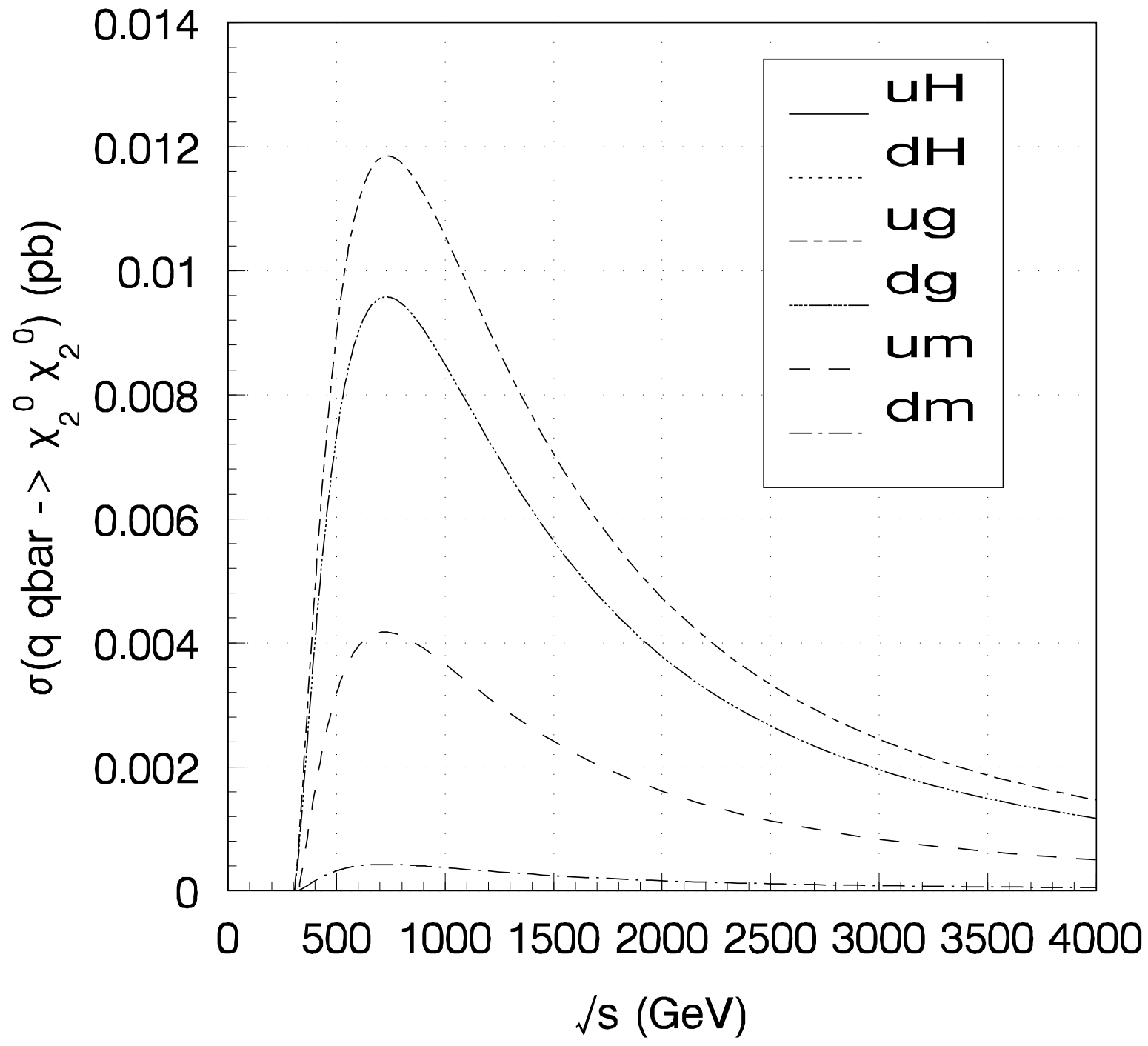


Fig.2(d)

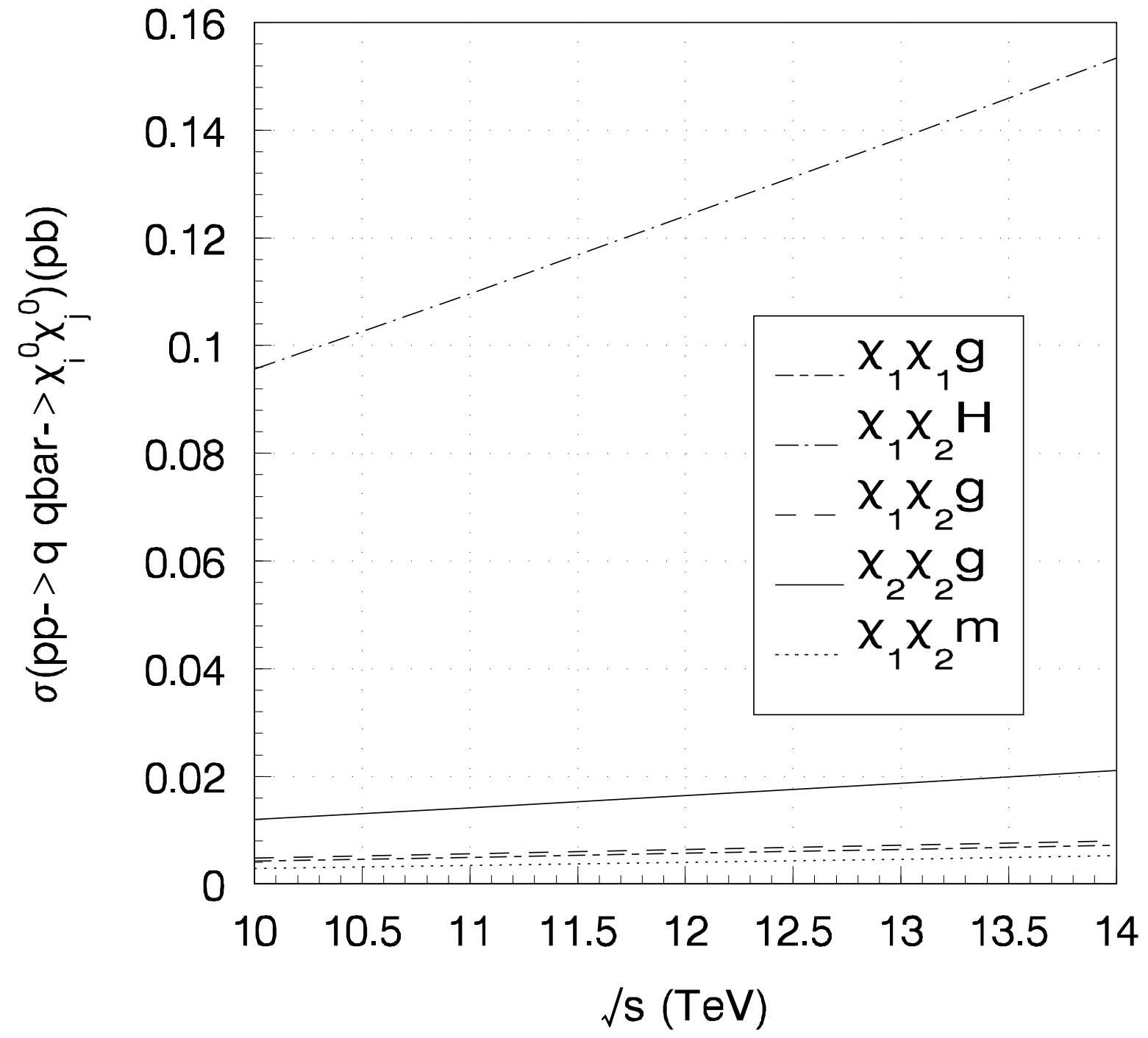


Fig.3(a)

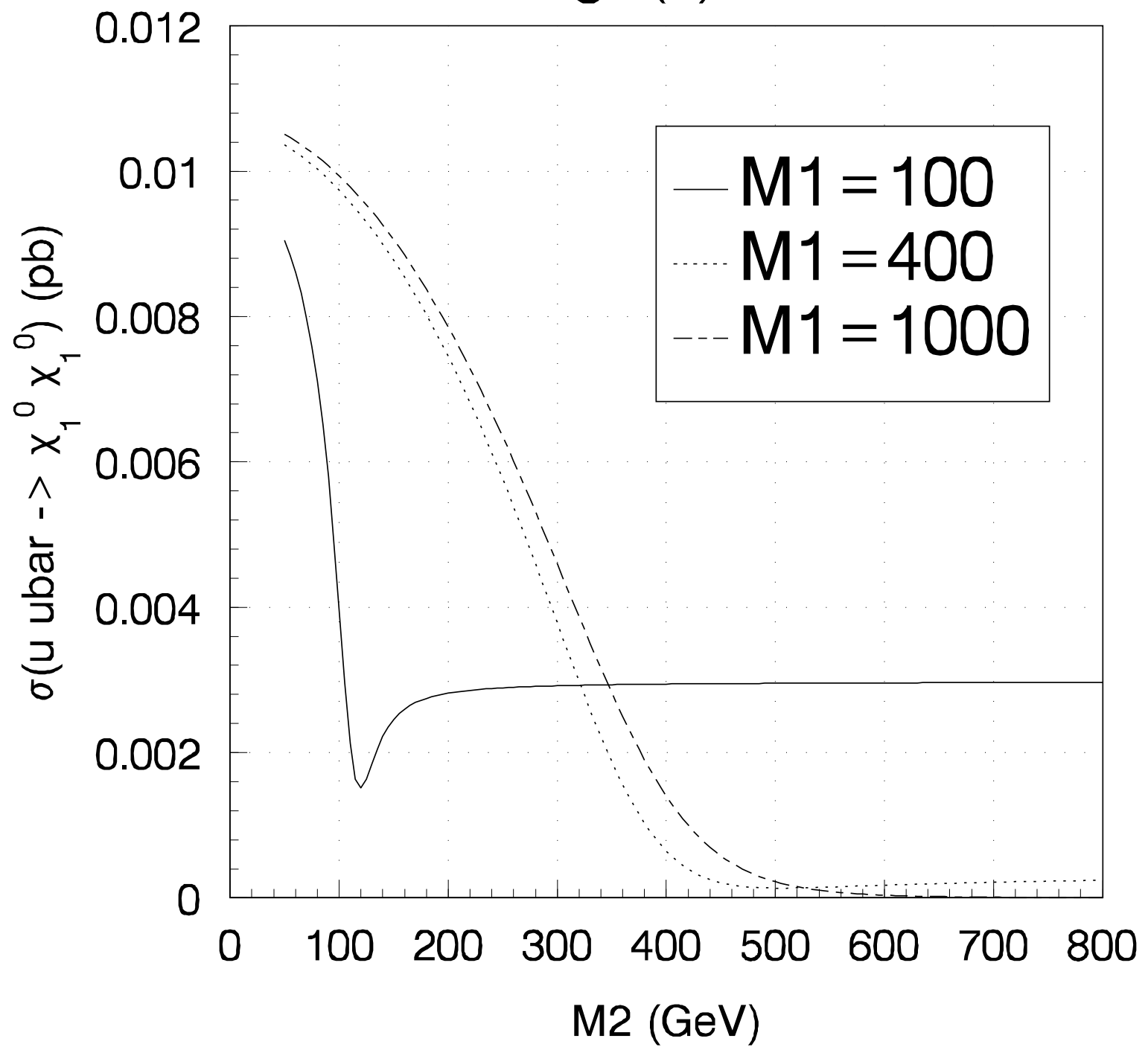


Fig.3(b)

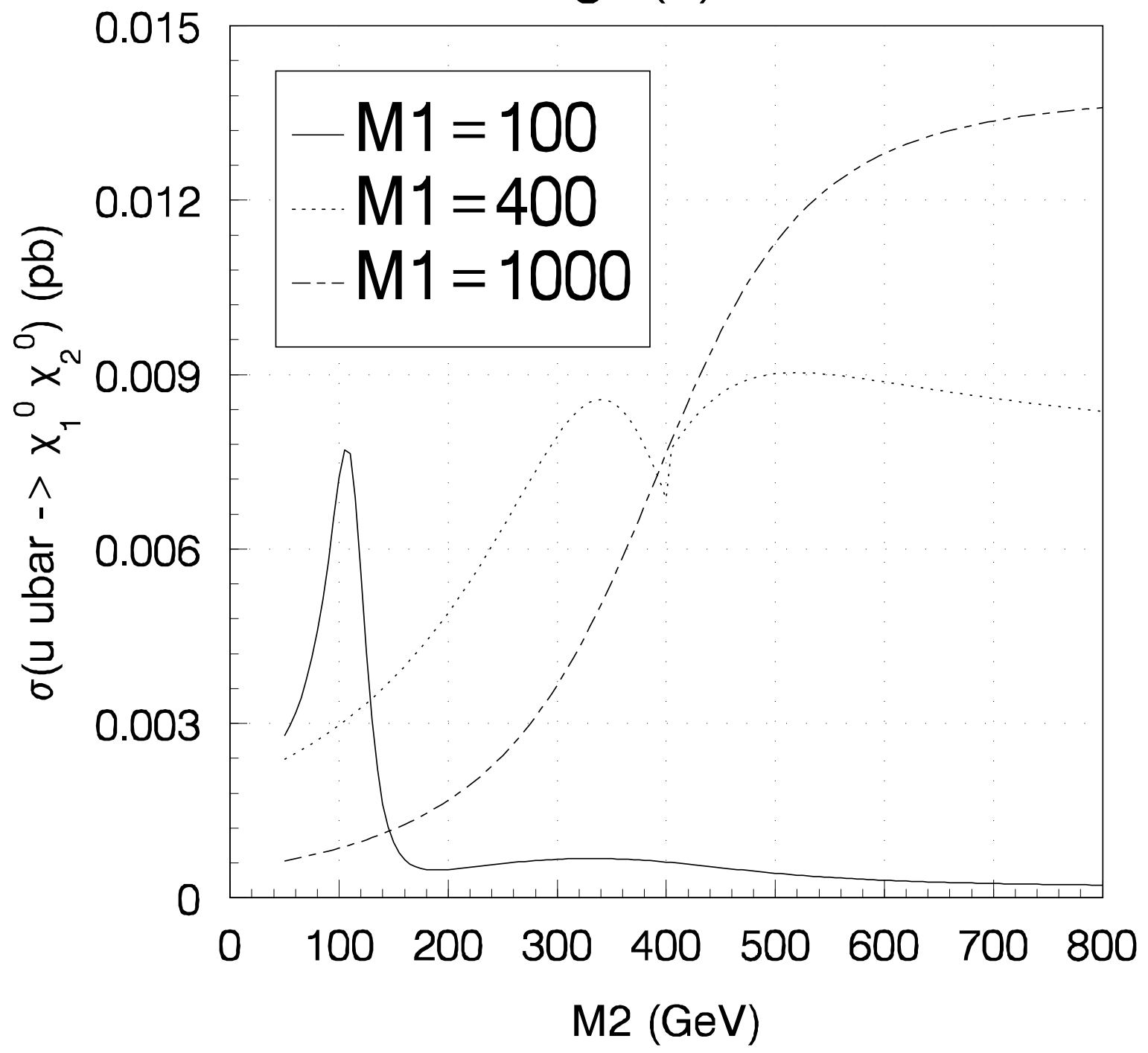


Fig.3(c)

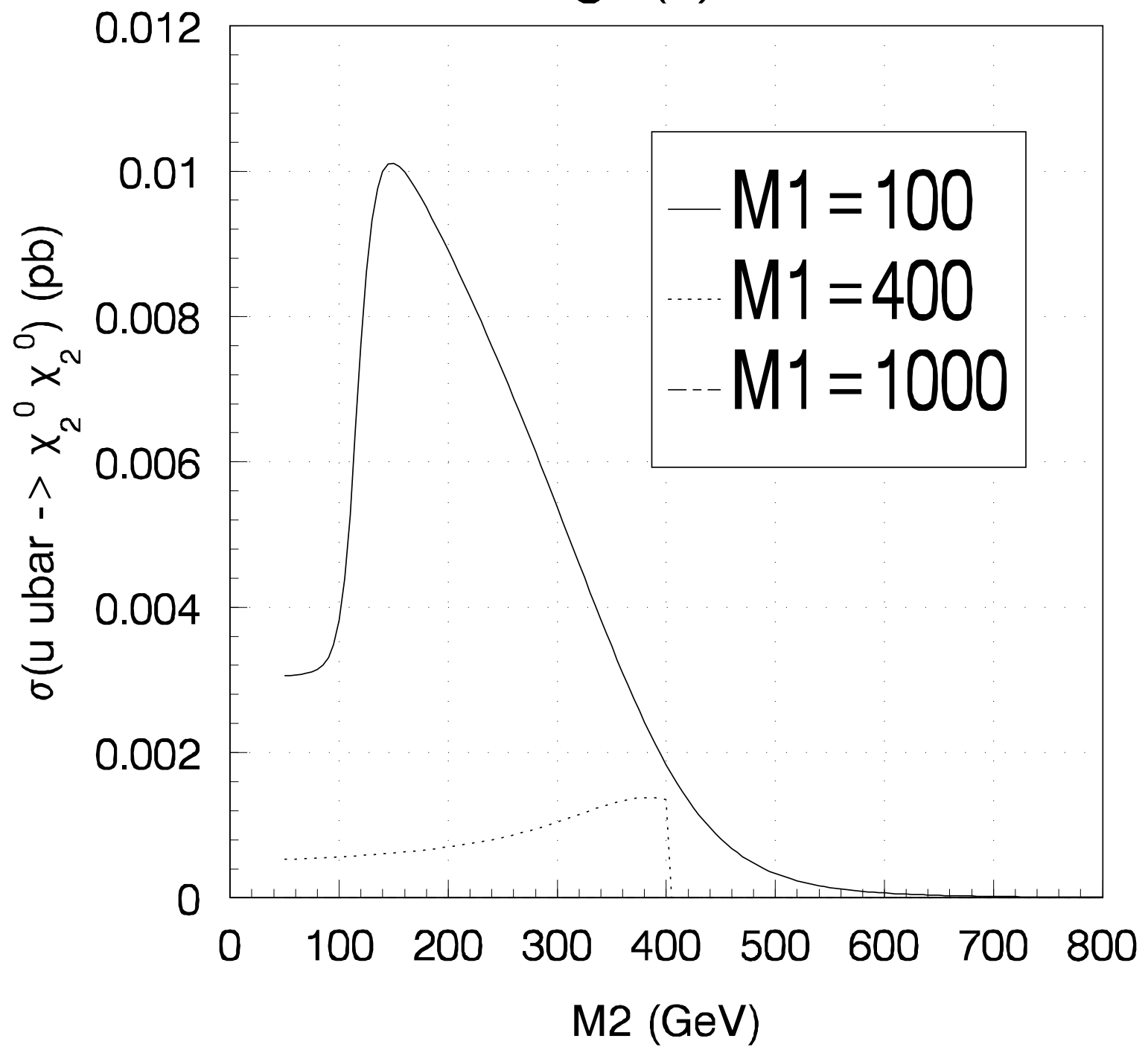

